\title{
Relation between the neutrino and quark mixing angles and grand unification
}

\author{
Martti Raidal \\ National Institute of Chemical Physics and Biophysics, \\ Ravala 10, Tallinn 10143, Estonia
}

\begin{abstract}
We argue that there exists simple relation between the quark and lepton mixings which supports the idea of grand unification and probes the underlying robust bi-maximal fermion mixing structure of still unknown flavor physics. In this framework the quark mixing matrix is a parameter matrix describing the deviation of neutrino mixing from exactly bi-maximal, predicting $\theta_{\text {sol }}+\theta_{C}=\pi / 4$, where $\theta_{C}$ is the Cabibbo angle, $\theta_{\text {atm }}+\theta_{23}^{C K M}=\pi / 4$ and $\theta_{13}^{M N S} \sim \theta_{13}^{C K M} \sim \mathcal{O}\left(\lambda^{3}\right)$, in a perfect agreement with experimental data. Both non-Abelian and Abelian flavor symmetries are needed for such a prediction to be realistic. An example flavor model capable to explain this flavor mixing pattern, and to induce the measured quark and lepton masses, is outlined.
\end{abstract}


Introduction. Despite of enormous experimental progress in neutrino 1] and quark physics in recent years, the origin of flavor remain a mystery. In the standard model the Yukawa couplings are free parameters to be fixed from experimental data. Grand unified theories (GUTs) 2, 3], which are supported by the unification of gauge couplings 4] in the minimal supersymmetric standard model (MSSM), predict relations between the quark and lepton Yukawa couplings at the unification scale. Although those predictions must be corrected in the minimal GUTs if all three generations of particles are considered, the idea of grand unification has been widely accepted. In the context of GUTs, the structure of Yukawa couplings has been most commonly derived from the Froggatt-Nielsen mechanism [5] of Abelian flavor symmetry breaking. This mechanism naturally predicts small mixing angles which are related to hierarchical fermion masses via $\theta_{i j} \sim \sqrt{m_{i} / m_{j}}, i<j$, in a reasonable agreement with the experimental data on the quark mixing matrix (CKM) [6, 7, $[$ ]].

This picture has been challenged by the discovery of almost bi-maximal neutrino mixing. If the smallness of neutrino masses is explained with the seesaw mechanism [9], hierarchical Yukawa couplings with small off-diagonal elements must produce large neutrino mixing angles. Although this is technically possible [10, 11, 12, 13, 14], it requires numerical fine tunings between Yukawa couplings of different generations [15]. In this context nonAbelian flavor symmetries, continuous or discrete, can be considered better candidates for explaining the systematics in the neutrino mixing matrix (MNS) 16]. However, even in non-Abelian flavor models some numerical coefficients must be fixed by hand in order to simultaneously satisfy [17] the exactly maximal atmospheric neutrino mixing, $\sin ^{2} 2 \theta_{\text {atm }}=$ $1.00 \pm 0.05$, large but not maximal solar neutrino mixing, $\tan ^{2} \theta_{\text {sol }}=0.41 \pm 0.05$, vanishing $\sin ^{2} 2 \theta_{13}^{M N S}=0 \pm 0.065$, and small Cabibbo angle $\theta_{C}$ [6] (or the Wolfenstein parameter $\lambda$ [8]), $\lambda=\sin \theta_{C}=0.22$. Although the deviation of the neutrino mixing matrix from bi-maximal has been parametrized [18], and the numerical correlation with the Cabibbo mixing has been pointed out [12], no physics explanation relating the quark and lepton mixings has been given so far.

In this Letter we show that there actually exists a simple relation between the quark and lepton mixings which provides a new experimental evidence for grand unification. We argue that at fundamental level the underlying non-Abelian flavor physics is robust and admits only vanishing or maximal mixing angles. Indeed, with the $\mathrm{SO}(3)$ or $\mathrm{SU}(2)$ flavor symmetry, and with the simplest superpotentials for flavons, this has been shown to be the case [19]. 
Because of GUT constraints for the fermion mixing matrices, the quark and lepton flavor mixings are related, predicting

$$
\begin{aligned}
& \theta_{C}+\theta_{\text {sol }}=\frac{\pi}{4}, \quad \theta_{23}^{C K M}+\theta_{\text {atm }}=\frac{\pi}{4}, \\
& \theta_{13}^{M N S} \sim \theta_{13}^{C K M} \sim \mathcal{O}\left(\lambda^{3}\right),
\end{aligned}
$$

in a good agreement with the experimental data (see (99)). Those predictions test experimentally the idea of grand unification, and, additionally, probe the fundamental properties of still unknown flavor physics. They allow to rule out the proposed idea in forthcoming neutrino oscillation experiments (for example if $\theta_{13}^{M N S}$ close to the present bound will be measured).

The resulting picture is simple and predictive. In the Wolfenstein parametrization [8], there is just one nontrivial parameter $\lambda$ characterizing both the deviation of the CKM matrix from diagonal matrix, and the deviation of the neutrino mixing matrix from exactly bi-maximal. The non-Abelian flavor symmetry implies singular $2 \times 2$ sub-structures for the Yukawa matrices and, consequently, a prediction of hierarchical fermion masses. Realistic masses for all the fermions should come from the additional Froggatt-Nielsen type mechanism of U(1) flavor symmetry breaking. Since the breaking of the non-Abelian flavor symmetry which generates mixing, and the Abelian flavor symmetry which generates light fermion masses are not related, it is possible to predict (11) and to generate the realistic fermion masses at the same time [19]. Although in this picture the Cabibbo angle is a parameter measuring an additional rotation, it is intriguing to argue that it is related to the breaking of the Abelian flavor symmetry. Such a model building is beyond the scope of this paper.

Flavor mixing and unification. We start with discussing how the bi-maximal fermion mixing, and the additional rotation by $\theta_{C}$, are consistent with the MSSM superpotential and the GUT relations for the Yukawa couplings. This follows by an example how such a framework can arise from the underlying flavor physics.

The superpotential of the MSSM with singlet (right-handed) heavy neutrinos is given by

$$
W=D^{c} Y_{d} Q H_{1}+U^{c} Y_{u} Q H_{2}+E^{c} Y_{e} L H_{1}+N^{c} Y_{\nu} L H_{2}+\frac{1}{2} N^{c} M N^{c},
$$

where the Yukawa matrices $Y$ are $3 \times 3$ matrices which can be diagonalized by bi-unitary transformations $Y^{D}=U^{\dagger} Y V$, where $V, U$ refer to the rotation of left- and right-chiral fields, respectively (for a symmetric matrix $Y, U=V^{*}$ ). There are two types of GUT relations 
between the Yukawa couplings of Eq.(2) often considered in literature. If the MSSM fermions are assigned into multiplets according to the $\mathrm{SU}(5)$ gauge group, the minimal unified model predicts

$$
Y_{e}=Y_{d}^{T}, \quad Y_{u}=Y_{u}^{T}
$$

However, SU(5) GUTs do not include right-chiral neutrinos. The second constraint, so called $\mathrm{SO}(10)$ relation [14], relates the up-type Yukawa couplings as

$$
Y_{\nu}=Y_{u}
$$

Although the comparison of down quark and charged lepton masses implies that the minimal GUT relation (31) has to be corrected [20, 21], let us assume in the beginning that both the relations (3), (41) hold. After that we show how the prediction (11) can follow from the SU(5) relation (3) alone. After presenting our basic results we show that (3), (44) are actually unnecessarily restrictive for us, and the light quark masses can be realistic without spoiling the prediction (11).

Integrating out the heavy singlet neutrinos from Eq.(2), the seesaw mechanism [9] induces the effective operator

$$
\frac{1}{2} \kappa L L H_{2} H_{2}
$$

which after the electroweak symmetry breaking generates masses for the active neutrinos as $m_{\nu}=\kappa v^{2}=Y_{\nu}^{T} M^{-1} Y_{\nu} v^{2}$. We recall that what is observed in the quark and neutrino experiments at low energies, the quark and neutrino mixing matrices $V_{C K M}$ and $V_{M N S}$, respectively, are given by

$$
V_{C K M}=V_{u}^{\dagger} V_{d}, \quad V_{M N S}=V_{e}^{\dagger} V_{\nu}
$$

The right-rotations are not directly observable in the present experiments. In the following we assume that the heavy singlet neutrino mass matrix $M$ does not introduce observable mixing effects into the light neutrino mass matrix. It is convenient to think of the mixing matrices $U, V$ as the sequence of three $2 \times 2$ rotations [22] [26],

$$
V, U=R\left(\theta_{23}\right) R\left(\theta_{13}\right) R\left(\theta_{12}\right) \text {. }
$$

Firstly, this allows us to simplify our discussion. Secondly, we argue that the underlying flavor physics actually generates a sequence of $2 \times 2$ rotations, thus Eq.(17) could correspond to the real situation in generating the flavor. 
We argue that the underlying flavor physics admits only vanishing or maximal mixing, and that the experimental data supports this view on flavor. The well known result [10] is that the SU(5) relations (3) allow the maximal atmospheric neutrino mixing and the (almost) vanishing third generation mixing in the $V_{C K M}$ to be consistent with (2) and (6). Consider the relevant $2 \times 2$ rotations by $\theta_{23}$. Choosing a basis in which $Y_{u}, Y_{\nu}$ are diagonal, and working with the precision up to first order in $\lambda, V_{C K M}=1$ implies $V_{d}=1$. Consequently, the maximal atmospheric mixing should come from the maximal (2-3) mixing in $V_{e}$, which according to (3) corresponds to the unobservable maximal right-mixing $U_{d}$ in the down quark sector.

The vanishing (to first order in $\lambda$ ) (1-3) mixing angles in $V_{C K M}$ and $V_{M N S}$ can be obtained trivially by setting $\theta_{13}=0$ in all the mixing matrices involved.

If we deal with the (1-2) mixing angles in the same way as we discussed the atmospheric neutrino mixing, we obtain exactly bi-maximal $V_{M N S}$ and diagonal $V_{C K M}$. However, this does not correspond to reality. In the $V_{C K M}$ the only sizable non-zero mixing angle is the Cabibbo angle, while in the neutrino sector the solar mixing angle is bounded to be nonmaximal by several sigmas, $\tan ^{2} \theta_{\text {sol }}=0.41 \pm 0.05$ [17]. It is intriguing that the deviation from the exact bi-maximal mixing in $V_{M N S}$, and the deviation from the unit matrix in $V_{C K M}$ are correlated: both are in $\theta_{12}$.

To make the $V_{C K M}$ realistic, let us take the previously described Yukawa matrices giving bi-maximal neutrino mixing and introduce into $Y_{u}$ an additional (1-2) rotation by the Cabibbo angle, $V_{C}$,

$$
Y_{u} \rightarrow V_{C}^{T} Y_{u} V_{C}
$$

This implies that $V_{C K M}=V_{C}$ in agreement with the experiment. However, because of the $\mathrm{SO}(10)$ GUT relation (4), the same rotation by $V_{C}$ takes also place in $Y_{\nu}$. This, according to Eq.(6), rotates $V_{M N S}$ into an opposite direction and decreases the solar mixing angle by the Cabibbo angle, $\theta_{\text {sol }}=\pi / 4-\theta_{C}$. Thus the relation between the quark and neutrino mixing comes from the GUT relation (4). Let us see what experimental data tells about this relation. While $\theta_{C}^{e x p}=12.7^{\circ}$ with small errors, $\tan ^{2} \theta_{\text {sol }}=0.41 \pm 0.05$ implies $\theta_{s o l}^{e x p}=32.6^{\circ} \pm 1.6^{\circ}$. Thus,

$$
\theta_{\text {sol }}^{\text {exp }}+\theta_{C}^{e x p}=45.3^{\circ} \pm 1.6^{\circ} \quad(1 \sigma),
$$

in a perfect agreement with the prediction. We recall that, because of tiny first generation quark Yukawa couplings, $\theta_{C}$ practically does not run when evaluating from $M_{G U T}$ to low 
energies. For normally hierarchical neutrinos predicted by GUTs, $m_{\nu_{1}} \ll m_{\nu_{2}} \ll m_{\nu_{3}}$, this is also true for $\theta_{\text {sol }}$. Therefore the prediction is expected to hold also at low scale. However, in more general case, for example for degenerate light neutrinos [24], the renormalization effects might be important.

So far we have used both the $\mathrm{SU}(5)$ and $\mathrm{SO}(10)$ GUT relations to derive the prediction (11). However, (11) can also follow from the SU(5) GUT constraint alone, with the additional assumption that the phenomenological rotation by the $V_{C K M}$ is left-right symmetric (this is automatic for the symmetric $Y_{u}=Y_{\nu}$ ). Indeed, to zeroth order in $\lambda$ we have no left rotations and maximal right rotations diagonalizing $Y_{d}$, and vice verse for $Y_{e}$. In this basis $\left(Y_{u}, Y_{\nu}\right.$ are diagonal) we may introduce the corrections to order $\lambda$ as

$$
Y_{d} \rightarrow V_{C} Y_{d} V_{C}^{\dagger}
$$

instead of (8), which creates non-diagonal $V_{C K M}$ and decreases the maximal right-rotation in $U_{d}$ by $\theta_{C}$. Due to the $\mathrm{SU}(5)$ GUT relation (3), the rotation (10) affects also $V_{M N S}$ and implies $\theta_{\text {sol }}=\pi / 4-\theta_{C}$. Again, the same result is obtained as before. This framework is simpler than the previous one since only the SU(5) GUT constraints are involved. However, the equality of left and right rotations in (10) is an assumption replacing (41).

Extending our discussion beyond the first order in $\lambda$ is straightforward. Obviously the prediction $\theta_{\text {atm }}+\theta_{23}^{C K M}=\pi / 4$ holds, just the smallness of $\theta_{23}^{C K M} \approx \lambda^{2}$ does not allow to test the deviation of $\theta_{\text {atm }}$ from the maximal. The importance of going beyond the first order in $\lambda$ is in the prediction for $\theta_{13}^{M N S}$ which should be non-zero in order to see CP violation in the neutrino sector. Naturally we expect (up to renormalization corrections) $\theta_{13}^{M N S} \sim \theta_{13}^{C K M} \sim \mathcal{O}\left(\lambda^{3}\right)$ which is, unfortunately, too small for generating observable $\mathrm{CP}$ violating effects in the presently planned oscillation experiments.

We know that at least one of the simplest GUT relations, $Y_{e}=Y_{d}^{T}$, must be corrected. However, for obtaining our results we need that only the particle mixing, which comes from the breaking on some non-Abelian flavor symmetry, must reflect the GUT structure discussed so far. The masses of light quarks and leptons, which should come from the breaking of additional U(1) flavor symmetry (otherwise the light generations remain massless), can naturally differ from each other. To put it in another way, the flavor physics inducing (2) "knows" the underlying unification structure, but after the flavor symmetry breaking this is reflected only in the fermion mixing and not in the eigenvalues of the Yukawa matrices. 
Therefore the GUT conditions (3) and (4) are actually unnecessarily restrictive. For our results to be correct, we need that the diagonalizing matrices follow the GUT relations, and/or that the additional rotation by $V_{C K M}$ is left-right symmetric. The eigenvalues (quark masses) can be different (although the constraint $Y_{u}=Y_{\nu}$ is still allowed by experimental data [14]). In the following we show that this is exactly the picture what one expects to get from a simple non-Abelian flavor model.

Non-Abelian flavor model. To exemplify the ideas presented so far we need to present a model which generates two $2 \times 2$ maximal mixings from the breaking of non-Abelian flavor symmetry, and in which fermion masses and the mixing are not directly related to each other. Such a model (which requires some modifications) is presented in Ref. [19]. To sketch the ideas developed in [19], let us assume that the underlying flavor physics is based on $\mathrm{SO}(3)$ or $\mathrm{SU}(2)$ flavor symmetry. Let us first consider two generations of fermions (second and third) which couple to flavons $\phi$ via

$$
\begin{aligned}
W= & \left(E^{c} \phi_{E}\right)\left(L \phi_{1 L}\right) H_{1}+\left(D^{c} \phi_{D}\right)\left(Q \phi_{1 Q}\right) H_{1}+\left(U^{c} \phi_{U}\right)\left(Q \phi_{2 Q}\right) H_{2} \\
& +\left(N^{c} \phi_{N}\right)\left(L \phi_{2 L}\right) H_{2}+\frac{1}{2}\left(N^{c} \phi_{N}\right) M\left(N^{c} \phi_{N}\right) .
\end{aligned}
$$

In front of each term we implicitly assume a $\mathcal{O}\left(1 / \Lambda^{2}\right)$ coefficient, where $\Lambda$ is the flavor breaking scale. We assume $\Lambda$ to be close to $M_{G U T}$ so that the flavon-mediated nonstandard interactions do not affect our numerical results. We assume that the light neutrino masses come only from the seesaw mechanism and the flavor physics itself does not generate additional effective operator (5) so that hierarchical neutrino masses can be generated (this is not the case in [19] which considered degenerate neutrino masses). Degeneracy of light neutrinos in this context implies the $\mathrm{U}(1)$ breaking parameter of order unity.

It has been shown in [19] that, with the simplest superpotentials for the flavon fields, after symmetry breaking the flavons acquire two types of vacuum expectation values (vevs) (writing just schematically, up to coefficients of order $\Lambda$ )

$$
\left(\begin{array}{l}
0 \\
1
\end{array}\right) \quad \text { or } \quad\left(\begin{array}{l}
1 \\
1
\end{array}\right) \text {. }
$$

This is a robust prediction, any deviation from this vev structure requires considerably more sophisticated model building. Substituting those vevs into Eq.(11), one gets the Yukawa 
matrices of the types

$$
\left(\begin{array}{ll}
0 & 0 \\
0 & 1
\end{array}\right) \quad \text { or } \quad\left(\begin{array}{ll}
0 & 1 \\
0 & 1
\end{array}\right) \quad \text { or } \quad\left(\begin{array}{ll}
1 & 1 \\
1 & 1
\end{array}\right) \text {. }
$$

The predictions are clear: $(i)$ fermion masses must be hierarchical because one of the eigenvalues is always vanishing [25]; (ii) there are only vanishing or maximal flavor mixing, depending on the corresponding flavon vev. For example, the maximal atmospheric neutrino mixing and the vanishingly small $\theta_{23}$ in the CKM matrix require

$$
\begin{array}{r}
\left\langle\phi_{1 Q}\right\rangle=\left\langle\phi_{2 Q}\right\rangle=\left\langle\phi_{U}\right\rangle=\left\langle\phi_{2 L}\right\rangle=\left\langle\phi_{E}\right\rangle=\left\langle\phi_{N}\right\rangle=\left(\begin{array}{l}
0 \\
1
\end{array}\right), \\
\left\langle\phi_{1 L}\right\rangle=\left\langle\phi_{D}\right\rangle=\left(\begin{array}{l}
1 \\
1
\end{array}\right) .
\end{array}
$$

This produces particle mixing matrices $U, V$ in agreement with the GUT relations (3), (4). Therefore Eq.(14), (15) can be considered to be GUT constraints for the non-Abelian flavor breaking. However, the magnitude of Yukawa couplings themselves depends on the numerical coefficients in (11), and need not to follow the minimal GUT relations exactly.

This is how Eq.(11) generates just one $2 \times 2$ rotation $\theta_{23}$ in each $U, V$ of Eq.(17). In order to generate also the maximal (1-2) mixing, one must work with three fermion generations and to include additional superpotential terms for light generations into Eq.(11). To give small masses to the first and second generation fermions, there must be additional Froggatt-Nielsen type coefficients weighting those terms. Details for the relevant flavon superpotentials can be found in [19]. As a result, exactly bi-maximal mixing with the mixing matrices consistent with the GUT relations can be produced. The additional rotation by the CKM matrix via (8) or (10) should occur from the mechanism beyond this model. Thus $V_{C K M}$ should be considered as a phenomenological parameter matrix.

Before concluding let us emphasize that the ideas presented here rely on several untested assumptions such as small neutrino renormalization effects, absence of non-standard interactions, high flavour and seesaw scales etc. Although those requirements, in particular the one of high flavour breaking scale, are natural in the GUT context, they can be proven wrong in new experiments and induce important new observable effects. Degeneracy of light neutrinos in this scheme implies $\mathrm{U}(1)$ breaking parameters of order unity, and important renormalization effects. In those cases the results of this work should be reconsidered. 
Conclusions. We argue that the (yet unknown) underlying non-Abelian flavor physics implies exactly bi-maximal particle mixing structure in the fermion sector, and that $V_{C K M}$ measures the deviation of $V_{M N S}$ from being exactly bi-maximal. Thus, in the Wolfenstein parametrization, $\lambda$ is the single parameter characterizing the non-triviality of particle mixing both in the quark and lepton sector. We predict $\theta_{\text {sol }}+\theta_{C}=\pi / 4, \theta_{\text {atm }}+\theta_{23}^{C K M}=\pi / 4$ and $\theta_{13}^{M N S} \sim \theta_{13}^{C K M} \sim \mathcal{O}\left(\lambda^{3}\right)$, in a good agreement with the experimental data (9). Observable deviations from those predictions, in particular large $\theta_{13}^{M N S}$, allow to test the proposed scheme in the future neutrino experiments. This prediction can follow from the SU(5) (or $\mathrm{SU}(5)$ and $\mathrm{SO}(10)$ ) type GUT constraints for the fermion mixing matrices, and from the structure of $V_{C K M}$ and $V_{M N S}$ in (6). It can be considered to be $(i)$ a new experimental evidence for the idea of grand unification; (ii) a probe for underlying bi-maximality of the fermion mixing. Additionally, because of (almost) vanishing $2 \times 2$ sub-determinants of all the Yukawa matrices, this picture predicts hierarchical fermion masses in agreement with observations. This pattern requires both the non-Abelian flavor symmetry breaking (which generates mixing) and the additional Abelian flavor symmetry breaking (which generates masses for light generations). Based on [19], we have given an example how such a flavor structure could arise, and how it can be consistent with the observed light quark and lepton masses (yet predicting (10)).

Acknowledgment. I would like to thank Gian Giudice and Graham Ross for discussions and Alessandro Strumia for providing the results of his latest fit to neutrino oscillation data. This work was supported by the ESF Grants 5135 and 5935, by the EC MC contract MERG-CT-2003-503626, and by the Ministry of Education and Research of the Republic of Estonia.

[1] M. H. Ahn et al. [K2K Collaboration], Phys. Rev. Lett. 90, 041801 (2003) arXiv:hep-ex/0212007;

K. Eguchi et al. [KamLAND Collaboration], Phys. Rev. Lett. 90, 021802 (2003) arXiv:hep-ex/0212021;

S. N. Ahmed et al. [SNO Collaboration], arXiv:nucl-ex/0309004.

[2] H. Georgi and S. L. Glashow, Phys. Rev. Lett. 32, 438 (1974). 
[3] H. Georgi, Particles and Fields, Proc. of the APS Div. of Particles and Fields, ed. C. Carlson, p. $575(1975)$;

H. Fritzsch and P. Minkowski, Annals Phys. 93, 193 (1975).

[4] U. Amaldi, W. de Boer and H. Furstenau, Phys. Lett. B 260, 447 (1991);

J. R. Ellis, S. Kelley and D. V. Nanopoulos, Phys. Lett. B 260, 131 (1991);

P. Langacker and M. x. Luo, Phys. Rev. D 44, 817 (1991).

[5] C. D. Froggatt and H. B. Nielsen, Nucl. Phys. B 147, 277 (1979).

[6] N. Cabibbo, Phys. Rev. Lett. 10, 531 (1963).

[7] M. Kobayashi and T. Maskawa, Prog. Theor. Phys. 49, 652 (1973).

[8] L. Wolfenstein, Phys. Rev. Lett. 51, 1945 (1983).

[9] M. Gell-Mann, P. Ramond and R. Slansky, Proceedings of the Supergravity Stony Brook Workshop, New York, 1979, eds. P. Van Nieuwenhuizen and D. Freedman (North-Holland, Amsterdam); T. Yanagida, Proceedings of the Workshop on Unified Theories and Baryon Number in the Universe, Tsukuba, Japan 1979 (eds. A. Sawada and A. Sugamoto, KEK Report No. 79-18, Tsukuba);

R. Mohapatra and G. Senjanovic, Phys. Rev. Lett. 44, 912 (1980).

[10] G. Altarelli and F. Feruglio, Phys. Rept. 320, 295 (1999); G. Altarelli and F. Feruglio, arXiv:hep-ph/0306265.

[11] M. C. Gonzalez-Garcia and Y. Nir, Rev. Mod. Phys. 75, 345 (2003) arXiv:hep-ph/0202058.

[12] A. Y. Smirnov, arXiv:hep-ph/0402264.

[13] S. F. King, Phys. Lett. B 439, 350 (1998) arXiv:hep-ph/9806440; Nucl. Phys. B 562, 57 (1999) arXiv:hep-ph/9904210.

[14] W. Buchmuller and D. Wyler, Phys. Lett. B 521, 291 (2001) arXiv:hep-ph/0108216.

[15] For a criticism and references see, e.g., S. L. Chen, M. Frigerio and E. Ma, arXiv:hep-ph/0404084.

[16] Z. Maki, M. Nakagawa and S. Sakata, Prog. Theor. Phys. 28, 870 (1962).

[17] M. Maltoni, T. Schwetz, M. A. Tortola and J. W. F. Valle, arXiv:hep-ph/0405172

J. N. Bahcall and C. Pena-Garay, arXiv:hep-ph/0404061;

P. Creminelli, G. Signorelli and A. Strumia, JHEP 0105, 052 (2001) arXiv:hep-ph/0102234 v5], A. Strumia, private communication.

[18] W. Rodejohann, Phys. Rev. D 69, 033005 (2004) arXiv:hep-ph/0309249. 
[19] R. Barbieri, L. J. Hall, G. L. Kane and G. G. Ross, arXiv:hep-ph/9901228.

[20] H. Georgi and C. Jarlskog, Phys. Lett. B 86, 297 (1979).

[21] G. Altarelli, F. Feruglio and I. Masina, JHEP 0011, 040 (2000) arXiv:hep-ph/0007254.

[22] J. Schechter and J. W. F. Valle, Phys. Rev. D 21, 309 (1980).

[23] J. Schechter and J. W. F. Valle, Phys. Rev. D 22, 2227 (1980).

[24] See for example, K. S. Babu, E. Ma and J. W. F. Valle, Phys. Lett. B 552, 207 (2003) arXiv:hep-ph/0206292.

[25] A different realization, and references for this idea can be found,e.g., in, I. Dorsner and A. Y. Smirnov, arXiv:hep-ph/0403305.

[26] Counting of physical phases [23], which is different for Dirac and Majorana neutrinos, does not affect our discussion here. 